\title{
Surface Channeling in Aberration-Corrected STEM of Nanostructures
}

\author{
Jingyue (Jimmy) Liu* and Lawrence F. Allard** \\ * Center for Nanoscience, Department of Physics and Astronomy, Department of Chemistry and \\ Biochemistry, UM-St. Louis, One University Boulevard, St. Louis, MO 63121 \\ ** Materials Science \& Tech. Division, Oak Ridge National Laboratory, Oak Ridge, TN 37831
}

Sub-Ångström resolution imaging and atomic resolution spectroscopy in aberration-corrected STEM are becoming routinely available, providing information on crystal structure, defect structure, elemental composition, and electronic structure with a sensitivity of, or approaching, individual atoms. To quantitatively interpret image intensities, however, requires a better understanding of the origin of the collected signals. We report here observation of anomalous intensity enhancement in sub-Ångström resolution HAADF images of surfaces and interfaces of $\mathrm{ZnO}$ nanostructures. We propose that the significant increase of the collected HAADF signal of a row of atom columns close to the crystal surface originates from a surface-resonance channeling effect [1].

The $\mathrm{ZnO}$ nanobelts used in these experiments were fabricated by a thermal evaporationcondensation method in a high-temperature tube furnace. Each nanobelt is a single crystal with relatively uniform thickness and some nanobelts possess atomically flat and clean surfaces. A JEOL 2200FS FEG STEM/TEM with a hexapole aberration corrector (CEOS GmbH, Heidelberg, Ger.) for the probe-forming optics, providing a nominal resolution of $0.07 \mathrm{~nm}$ in HAADF imaging mode, was used to obtain the HAADF images. The HAADF detector was operated with inner and outer collection semi-angles of $100 \mathrm{mrad}$ and $170 \mathrm{mrad}$, respectively.

Figures 1a and 1b show, respectively, a HAADF image and the corresponding BF STEM image of a $\mathrm{ZnO}$ nanobelt, oriented along the [11-20] zone axis. The computed diffractogram is shown in Fig. 1b. The amorphous material, clearly revealed in Fig. 1b, originated from the buildup of electron beam-induced contamination. Even with the presence of a thick layer of amorphous material, atomic resolution images were obtained as is clearly shown in the inset of Fig. 1a. In addition to revealing the atomic arrangement of the $\mathrm{Zn}$ columns, the most striking feature in the HAADF images is the bright row of atom columns (hereafter, 'bright atom row'), running along the $\mathrm{ZnO}$ [-1100] direction, close to the $\mathrm{ZnO}$ (0001) surface; the BF STEM image shows a dark band corresponding to the same near-surface atomic columns. To quantify the intensity enhancement of the bright atom row, an intensity profile across the $\mathrm{ZnO}(0001)$ lattice fringes within the rectangular box in Fig. 1a, projected along the $\mathrm{ZnO}$ [-1100] direction, is displayed in Fig. 1c (Only a portion of the intensity profile is shown). The intensity enhancement of the bright atom row close to the (0001) surface is significant; the fringe visibility of this row was estimated to be about 2.5 times that of the lattice fringes inside the $\mathrm{ZnO}$ nanobelts. The spacing between the bright atom row and the next row of $\mathrm{Zn}$ atoms within the $\mathrm{ZnO}$ crystal was measured to be approximately $0.30 \mathrm{~nm}$, much larger than the $\mathrm{ZnO}$ bulk lattice spacing of $0.26 \mathrm{~nm}$. The high magnification HAADF image shown in the inset of Fig. 1a reveals that the bright, near-surface atom plane does not represent the outmost surface atoms of the $\mathrm{ZnO}(0001)$ surface. There is at least one layer, and in some places probably two layers, of $\mathrm{Zn}$ atoms beyond this bright atomic plane. These layers, however, possess much lower intensities than those of the bulk $\mathrm{Zn}$ layers, suggesting that the $\mathrm{ZnO}$ (0001) surface has an incomplete growth of the outer-most surface layer. The bright atom row represents the first complete $\mathrm{ZnO}$ (0001) layer near the surface. These observations and a series of other experiments suggest that a surface-resonance channeling 
effect may play a major role in determining the observed anomaly of intensity enhancement of HAADF images of structurally perfect surfaces [1]. A better understanding of the effect of surface/interface channeling on $\mathrm{HAADF} / \mathrm{ADF}$ image contrast may have implications for the quantitative interpretation of those images and may provide new routes to utilization of this technique to study surface structures with sub-Ångström resolution [2].

\section{References}

1 J.M. Cowley, Ultramicroscopy 27 (1989) 319.

2 This research is supported by the University of Missouri-St. Louis; the electron microscopy work was conducted at the Oak Ridge National Laboratory's High Temperature Materials Laboratory, sponsored by the U.S. DOE, Office of Energy Efficiency \& Renewable Energy, Vehicle Technologies Program.
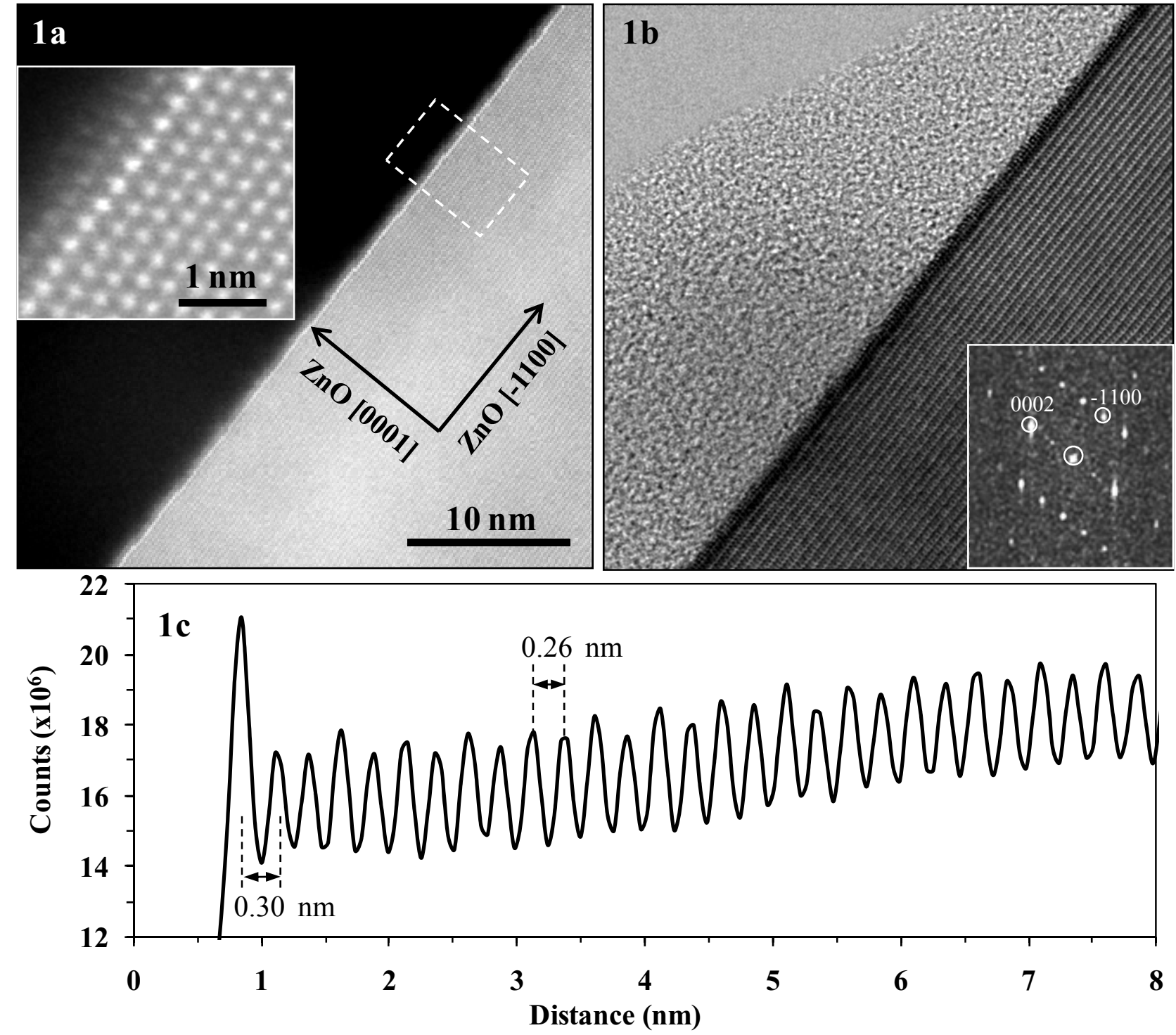

FIG. 1. a) HAADF image and b) the corresponding BF STEM image of a ZnO nanobelt. Magnified HAADF image is shown in inset of Fig. 1a and the computed diffractogram is shown in inset of Fig. 1b. An intensity profile across the $\mathrm{ZnO}$ (0001) lattice fringes within the rectangular box in Fig. 1a, projected along the $\mathrm{ZnO}[-1100]$ direction, is displayed in Fig. 1c. 